\title{
Efeito da Inversão dos Turnos de Trabalho Sobre Capacidade Aeróbia e Respostas Cardiovasculares ao Esforço Máximo
}

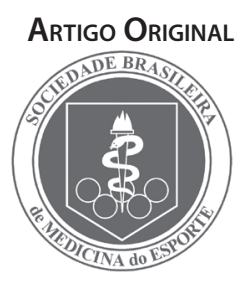

\section{Effect of the Work Shift Reversal on the Aerobic Capacity and Cardiovascular Responses to Maximal Exercise}

Anna Myrna Jaguaribe de Lima Cláudia Maria Vieira Soares ${ }^{2}$ Alexandre Oscar Soares de Souza ${ }^{3}$

1. Faculdade Integrada do Recife (FIR), Recife-PE, Brasil e Fisioterapia da Faculdade do Agreste de Pernambuco (FAAPE), Caruaru-PE, Brasil.

2. Reabily, Recife-PE, Brasil 3. Faculdade do Agreste de Pernambuco (FAAPE), Caruaru-PE, Brasil.

\section{Endereço para correspondência:} Rua José Armando Machado, N-91

- Apto-304 - Boa Viagem

Recife - PE - CEP 51130-170

Email: annamyrna@uol.com.br

Submetido em 30/06/2006 Versão final recebida em 03/01/2008 Aceito em 04/01/2008

\begin{abstract}
RESUMO
Os distúrbios do sono e alterações associadas atingem grande parte da população que trabalha no turno noturno, afetando a sua qualidade de vida. O objetivo do presente trabalho foi comparar a capacidade aeróbia e as respostas cardiovasculares ao exercício máximo em indivíduos com ciclo sono vigília fisiológico e com inversão dos turnos de trabalho. Foram analisados 18 indivíduos do sexo masculino, sedentários, com idade entre 23-35 anos, divididos em 2 grupos: a) grupo controle, formado por estudantes ( $n=9)$ e b) grupo experimental, composto por controladores de tráfego aéreo que trabalhavam com inversão dos turnos de trabalho $(n=9)$. Para a medida da capacidade aeróbia, foi determinado o VO máx. por meio do analisador de gases metabólicos (VO-2000, Aerosport, Medgraphics). Para o teste de esforço máximo foi realizado o protocolo de rampa em esteira (Millenniun ATL - Inbramed) e as respostas cardiovasculares (FC, PAS e PAD) foram verificadas antes e após a realização do exercício. De acordo com os resultados, o grupo experimental apresentou valores inferiores de FC no repouso ( $79,8 \pm 11,5 \mathrm{bpm}$ vs. $70,3 \pm 3,8 \mathrm{bpm})$, no $5^{\circ}(112,7 \pm 15,1$ bpm vs. $98,7 \pm 6,3 \mathrm{bpm})$ e no $7^{\circ}$ minuto $(108,7 \pm 16,6 \mathrm{bpm}$ vs. $93,9 \pm 6,8 \mathrm{bpm})$ de recuperação. Quanto à PAS, foram observados valores superiores durante o repouso $(110,0 \pm 11,2 \mathrm{mmHg}$ vs. $104,0 \pm 5,7 \mathrm{mmHg})$, nos indivíduos do grupo controle. Já a PAD, mostrou níveis superiores no $5^{\circ}$ minuto da recuperação no grupo experimental $(67,0 \pm 4,4 \mathrm{mmHg}$ vs. $58,9 \pm 6,0 \mathrm{mmHg})$. Por fim, foram verificados valores superiores de $\mathrm{VO}_{2}$ máx. para os indivíduos do grupo controle $(58,9 \pm 6,1 \mathrm{ml} / \mathrm{kg} / \mathrm{min})$ em relação ao experimental $(53,7$ $\pm 2,5 \mathrm{ml} / \mathrm{kg} / \mathrm{min}$ ). Desta forma, podemos concluir que a inversão nos turnos de trabalho, afetando o ciclo sono-vigília, altera não apenas o ciclo circadiano das variáveis cardiovasculares no repouso e na recuperação do esforço, como também traz prejuízos à capacidade funcional, podendo comprometer o desempenho das atividades ocupacionais.
\end{abstract}

Palavras-chave: $\mathrm{VO}_{2}$ máx., variáveis cardiovasculares, controladores de vôo, exercício máximo.

\begin{abstract}
Sleep disturbs and associated alterations reach great part of the population which works at night, affecting their quality of life. The aim of the present study was to compare the aerobic capacity and the cardiovascular responses to maximal exercise in subjects with physiological sleep/wake cycle and with work shift inversion. 18 male subjects, aged 23-35 years, were assigned to either a shift-workers (Experimental; $n=9$ ) or a control group (Control; $n=9$ ). All the subjects underwent a treadmill exercise test in order to obtain the BP, HR behavior in the recovery period. Exhaled air was collected every 10 minutes for $\mathrm{VO}_{2}$ max. Shift-workers presented lower heart rate values at rest (79.8 $\pm 11.5 \mathrm{bpm}$ vs. $\left.70.3 \pm 3.8 \mathrm{bpm}\right)$, $5^{\text {th }}(112.7 \pm 15,1 \mathrm{bpm}$ vs. $98,7 \pm 6,3 \mathrm{bpm})$ and $7^{\text {th }}$ min. $(108.7 \pm 16.6 \mathrm{bpm}$ vs. $93.9 \pm 6.8 \mathrm{bpm})$ at recovery. SBP showed significant increased values in control group just at rest $(110.0 \pm 11.2 \mathrm{mmHg}$ vs. $104.0 \pm 5.7$ $\mathrm{mmHg})$. The control group presented lower values of DBP at the $5^{\text {th }} \mathrm{min} .(67.0 \pm 4.4 \mathrm{mmHg}$ vs. $58.9 \pm$ $6.0 \mathrm{mmHg})$ and higher values of $\mathrm{VO}_{2} \max .(58.9 \pm 6.1 \mathrm{ml} / \mathrm{kg} / \mathrm{min}$ vs. $53.7 \pm 2.5 \mathrm{ml} / \mathrm{kg} / \mathrm{min})$. We concluded that the alterations in the working shifts affect the circadian rhythms and the cardiovascular variables at rest and recovery periods of the exercise stress testing and can compromise the functional capacity of the subjects.
\end{abstract}

Keywords: $\mathrm{VO}_{2}$ max., cardiovascular variables, air traffic controllers, maximal exercise. 


\section{INTRODUÇÃO}

O sistema cardiovascular apresenta um ritmo circadiano, que pode ser afetado pelas alterações dos turnos de trabalho(1-2). Modificações fisiológicas, tais como: aumento da agregação plaquetária, da PA, da FC, da secreção de catecolaminas, do tônus simpático e dos níveis plasmáticos de cortisol apresentam maior incidência no período da manhã ${ }^{(3-5)}$. A inversão dos turnos de trabalho está associada também com diversos problemas de saúde como doenças cardiovasculares, desconforto gastrointestinal, distúrbios de reprodução e câncer de mama ${ }^{(6-7)}$

A fadiga após longos períodos de trabalho sem intervalos pode comprometer tanto o desempenho físico como mental dos trabalhadores em situação de inversão de turnos de trabalho. $O$ trabalho realiza-se numa fase de desativação psicossomática e na situação de descanso, provocando uma inversão dos ritmos biológicos e interferindo na dessincronização entre ciclo sono-vigília e ciclo dia/noite, gerando uma sintomatologia de distúrbios do sono, fadiga, alterações de humor, transtornos digestivos, neuropsicológicos, diminuição da motivação para o trabalho, sentimento de exclusão da vida social e perturbação da convivência familiar e da saúde, diminuindo os estímulos auditivos e visuais, sendo observada uma maior freqüência de acidentes principalmente no turno da noite ${ }^{(8-10)}$.

Especificamente sobre os controladores de vôo, além dos problemas ocasionados pela inversão dos turnos de trabalho, as tarefas múltiplas realizadas simultaneamente e com diferentes graus de complexidade, requerem maiores níveis de atenção desses indivíduos ${ }^{(11,12)}$.

Desta forma, o objetivo do presente trabalho foi comparar o comportamento das variáveis cardiovasculares e metabólicas em indivíduos com ciclo sono-vigília fisiológico e com inversão dos turnos de trabalho submetidos ao exercício aeróbio máximo.

\section{MÉTODOS}

\section{Amostra}

Foram analisados 18 indivíduos (n obtido por cálculo amostral), do sexo masculino, sedentários, com idade entre 23-35 anos, divididos em 2 grupos: a) grupo controle, formado por estudantes universitários com ciclo sono-vigília em padrão fisiológico $(n=9)$ e b) grupo experimental, composto por controladores de tráfego aéreo que trabalhavam com inversão dos turnos de trabalho e, pelo menos 3 vezes por semana, tinham seu horário de trabalho entre 22 hs e 3 hs ou 3hs-7hs ( $n=9$ ). Foram excluídos do estudo indivíduos fumantes e/ou que tinham ingerido álcool até 48hs antes do teste e/ou com patologias cardiopulmonares. Todos os participantes da pesquisa receberam orientações a respeito dos procedimentos adotados durante o protocolo experimental e assinaram um termo de consentimento para realização do estudo. Este estudo foi aprovado pelo comitê de ética do Hospital Agamenon Magalhães de acordo com a resolução 196/96.

\section{Teste de Esforço Máximo e Análise de Gases Metabólicos}

O teste de esforço máximo foi realizado no período da manhã (7hs às 10hs), numa temperatura $22-24^{\circ}, 55 \%$ de umidade e uma pressão atmosférica de $760 \mathrm{mmhg}$. Utilizamos a esteira Millenniun ATL - Ibramed, e o protocolo de Rampa, velocidade inicial de $3,0 \mathrm{~km} / \mathrm{h}$, velocidade final de $15 \mathrm{~km} / \mathrm{h}$, inclinação inicial de $0 \%$ e final de $5 \%$ e tempo máximo de duração do teste igual a 10min. Os controladores de vôo realizavam os teste de esforço sempre $24 \mathrm{~h}$ após terem trabalhado no período da noite (22:00-7:00).

Para a medida do $\mathrm{VO}_{2}$ máx. foi utilizado o analisador de gases metabólicos (VO-2000, Aerosport, Medgraphics, St. Paul, Minnesota), no qual as amostras gasosas eram coletadas a cada 10s durante o teste. Os critérios considerados para atingir $\mathrm{O} \mathrm{VO}_{2}$ máx. foram alcançar pelo menos 2 dos parâmetros a seguir: estabelecimento de platô da curva de consumo de $\mathrm{O}_{2}$ em relação à carga; razão das trocas gasosas $\geq 1,0$; exaustão física do indivíduo.

\section{Pressão Arterial}

A monitorização da PA foi feita antes e no período de recuperação $\left(1^{\circ}, 2^{\circ}, 5^{\circ}\right.$ e $7^{\circ}$ minuto) do teste de esforço máximo, utilizando-se do método auscultatório (tensiômetro marca Solidor e o estetoscópio Littman ${ }^{\circ}$.

\section{Freqüência Cardíaca}

Para a monitorização FC foi utilizado um eletrocardiógrafo Micromed, na derivação CM5, verificada antes, durante e na recuperação (1 ${ }^{\circ}$, $2^{\circ}, 5^{\circ}$ e $7^{\circ}$ minuto) do teste de esforço máximo.

\section{Análise Estatística}

Para verificação da homogeneidade da amostra e análise das variáveis cardiovasculares (FC, PAS, PAD) e do $\mathrm{VO}_{2}$ máx. antes e após o teste de esforço máximo, em indivíduos com inversão dos turnos de trabalho e em voluntários com ciclo sono-vigília fisiológico, utilizamos o Teste $t$ Student para amostras independentes. Os resultados mostrados como média \pm desvio padrão e foi considerado como nível de significância um $\mathrm{p}<0,05$.

\section{RESULTADOS}

Na tabela 1, podemos verificar os dados de idade, peso, altura e índice de massa corpórea de ambos os grupos, o que caracteriza a homogeneidade da amostra.

A tabela 2 demonstra o comportamento da FC, no repouso e na recuperação do exercício máximo em indivíduos com o ciclo sonovigília fisiológico e com inversão dos turnos de trabalho. Observamos valores menores na FC de repouso, no $5^{\circ}$ min e 70 min de recuperação nos indivíduos com ciclo sono-vigília com inversão dos turnos de trabalho.

Quando comparamos os grupos, controle e experimental, (Tabela 1) verificamos níveis inferiores na PAS de repouso. No entanto, não foram observadas diferenças significativas no período de recuperação.

No que diz respeito a PAD, verificamos valores menores no grupo controle apenas durante o $5^{\circ}$ min de recuperação (Tabela 1).

No grupo controle, o consumo máximo de $\mathrm{O}_{2}$ foi superior quando comparado ao grupo experimental (Figura 1).

\section{DISCUSSÃO}

Os processos fisiológicos são regidos por alterações cíclicas regulares que denominamos de ritmos biológicos. No presente estudo, analisamos o comportamento da capacidade aeróbia e das variáveis cardiovasculares em indivíduos com ciclo sono-vigília fisiológico e naqueles que tinham seu turno de trabalho invertido.

Tabela 1. Caracterização dos Dados da Amostra

\begin{tabular}{c|c|c|c|c}
\hline & Idade (anos) & Peso $(\mathbf{k g})$ & Altura $(\mathbf{m})$ & IMC $\left(\mathbf{k g} / \mathbf{m}^{2}\right)$ \\
\hline $\begin{array}{c}\text { Grupo } \\
\text { Controle }\end{array}$ & $25,9 \pm 4,3$ & $78 \pm 11,8$ & $1,76 \pm 0,01$ & $25 \pm 3,2$ \\
\hline $\begin{array}{c}\text { Grupo } \\
\text { Experimental }\end{array}$ & $29,4 \pm 3,4$ & $79,2 \pm 10,2$ & $1,74 \pm 0,06$ & $26,1 \pm 2,4$ \\
\hline
\end{tabular}


Tabela 2. FC, PAS e PAD, nos grupos controle e experimental, no repouso e na recuperação do exercício máximo

\begin{tabular}{|c|c|c|c|c|c|c|}
\hline & \multicolumn{2}{|c|}{ Freqüência Cardíaca (bpm) } & \multicolumn{2}{|c|}{ Pressão Arterial Sistólica (mmHg) } & \multicolumn{2}{|c|}{ Pressão Arterial Diastólica $(\mathrm{mmHg})$} \\
\hline & $\begin{array}{l}\text { Grupo } \\
\text { Controle }\end{array}$ & $\begin{array}{c}\text { Grupo } \\
\text { Experimental }\end{array}$ & $\begin{array}{l}\text { Grupo } \\
\text { Controle }\end{array}$ & $\begin{array}{c}\text { Grupo } \\
\text { Experimental }\end{array}$ & $\begin{array}{l}\text { Grupo } \\
\text { Controle }\end{array}$ & $\begin{array}{c}\text { Grupo } \\
\text { Experimental }\end{array}$ \\
\hline Repouso & $79,8 \pm 11,5$ & $70,3 \pm 3,8^{*}$ & $113,1 \pm 7,2$ & $104,3 \pm 5,7^{*}$ & $68,4 \pm 8,8$ & $71,2 \pm 2,2$ \\
\hline 1'Recuperação & $129,1 \pm 18,8$ & $116,0 \pm 5,6$ & $134,4 \pm 16,7$ & $131,8 \pm 7,2$ & $68,4 \pm 8,8$ & $64,1 \pm 5,6$ \\
\hline 2'Recuperação & $116,4 \pm 13,4$ & $107,2 \pm 5,7$ & $128,9 \pm 14,5$ & $118,2 \pm 6,1$ & $61,4 \pm 6,6$ & $65,9 \pm 5,1$ \\
\hline 5'Recuperação & $112,7 \pm 15,1$ & $98,7 \pm 6,3^{*}$ & $110,0 \pm 11,2$ & $110,9 \pm 5,7$ & $58,9 \pm 6,0$ & $67,0 \pm 4,4^{*}$ \\
\hline 7'Recuperação & $108,7 \pm 14,6$ & $93,9 \pm 6,8^{*}$ & $107,8 \pm 12,0$ & $108,7 \pm 3,6$ & $61,1 \pm 9,3$ & $67,9 \pm 6,0$ \\
\hline
\end{tabular}

$\mathrm{n}=18,{ }^{*}$ Diferença significativa: (C) Controle vs. (E) Experimental, $\mathrm{p}<0.05$

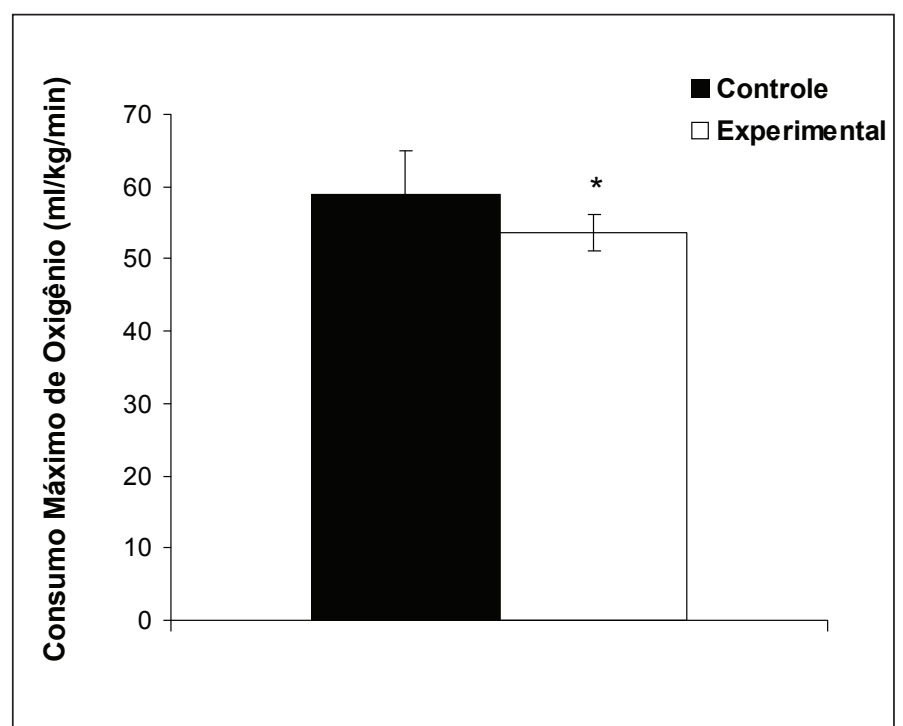

Figura 1. Consumo máximo de oxigênio em indivíduos com ciclo sono-vigília fisiológico (C) e com inversão dos turnos de trabalho (E). $\mathrm{n}=18,{ }^{*}$ Diferença significativa: (C) Controle vs. (E) Experimental, $\mathrm{p}<0.05$

White (2003) ${ }^{(13)}$ relatou a variação da FC de acordo com as horas do dia e observou níveis de FC maiores entre 6hs e 10hs da manhã. A inversão do ciclo sono-vigília causa uma modificação deste padrão, mostrando-se uma inversão, com valores maiores de FC encontrados no período das $22 \mathrm{hs}$ às $4 \mathrm{hs}$. Em nosso estudo, ao compararmos indivíduos com ciclo sono vigília fisiológico e com inversão dos turnos de trabalho, verificamos, no repouso e no $5^{\circ}$ e $7^{\circ}$ minutos de recuperação do exercício máximo, freqüências cardíacas inferiores nos indivíduos do grupo experimental (Tabela 2). Quanto à FC de repouso, estes achados são similares aos de Faria e Drummond (1982) apud Reilly, Atkinson e Waterhouse (2003) $)^{(14)}$ que relataram que a FC depende da hora do dia, sendo mais alta pela manhã do que à tarde. Isto pode ser explicado por uma alteração na modulação autonômica, que também é regulada pelo ritmo circadiano. Furlan et al. (2000)(3) estudaram o tônus autonômico através da análise da variabilidade da freqüência cardíaca em três turnos de trabalho diferentes e observaram que existia uma maior atividade simpática durante o período do trabalho, independente do turno em que era realizado. Eles relataram ainda que, em indivíduos que exerciam suas profissões em situação de ciclo sono vigília invertido, apesar da maior influência simpática, havia uma redução do estado de alerta, o que poderia comprometer o desempenho de suas atividades profissionais. Cohen (1980) ${ }^{(15)}$ estudou as respostas da FC durante o exercício máximo, pouco antes de chegar à exaustão e não verificou variação com a hora do dia, sugerindo a ausência de um ritmo na FCmáx, no entanto Reilly et al. (1982)(16) encontraram o ritmo na FC persistindo durante o exercício máximo.

No que diz respeito à PA, da mesma forma que a FC, também há um padrão fisiológico de flutuações diurnas, dependente dos ritmos circadianos, do ciclo de atividade e sono(17). Este controle acontece no núcleo supraquiasmático do hipotálamo, considerado o marcapasso dos ritmos, que controla a ritmicidade biológica endógena e a atividade autonômica e endócrina ${ }^{(18)}$. Pelo ritmo circadiano, a PA mostra níveis superiores no período diurno, permanecendo desta maneira de 4-6hs após o despertar. Em nosso estudo, verificamos níveis inferiores na PAS de repouso, no entanto, não foram observadas diferenças significativas na PAS no período de recuperação. Segundo Chau et al. (1989) ${ }^{(19)}$, quanto maior for o período de alteração do ciclo sono-vigília, maior será a alteração nos níveis pressóricos. Estas alterações podem ser atribuídas mais uma vez à modificação na modulação autonômica, com predomínio da atividade simpática nos períodos de alerta (durante o trabalho noturno) e aos ritmos de liberação das catecolaminas e cortisol, atividade do sistema reninaangiotensina, influenciando desta maneira, a pressão arterial. Esta variação na liberação hormonal e atividade do SCV têm uma relação direta com a maior ocorrência de eventos cardiovasculares como infartos e acidentes vasculares cerebrais no período da manhã, em indivíduos com ciclo sono vigília inalterado. No entanto, não existem estudos fazendo esta correlação com a alteração dos estados de sono e vigília.

Quanto à PAD, observamos diferença significativa entre os grupos apenas no $5^{\circ}$ minuto de recuperação (Tabela 2), mostrando níveis inferiores nos indivíduos com ciclo sono vigília invertido. Verificamos uma tendência a uma hipotensão pós-exercício mais pronunciada nestes indivíduos, o que pode ser justificado pela menor influência simpática também sobre os vasos, facilitando a ação vasodilatadora e reduzindo a resistência vascular periférica.

Por fim, no que diz respeito ao consumo máximo de oxigênio, encontramos no presente estudo valores significativamente maiores nos indivíduos do grupo controle em relação ao experimental (Figura 1). Deschenes et al. (1998)(20), relatam os efeitos do exercício, $\mathrm{O}_{2}$ e ventilação, não estão alterados através da cronobiologia, pois 
nenhuma dessas variáveis fisiológicas exibe respostas significantes no exercício. Reilly (1982)(16) relata que não foram encontradas variações para $\mathrm{CO}_{2}$ expirado $\left(\mathrm{VCO}_{2}\right)$ ou o quociente de trocas respiratórias durante o exercício, assim como Hildebrandt \& Strempel (1974)(21) que relatam que as respostas metabólicas ao exercício máximo não demonstram um ritmo circadiano e o $\mathrm{VO}_{2}$ máx é uma função estável, independentemente da hora do dia em que foi medida. Podemos justificar nossos achados não pela variação circadiana dos níveis de consumo máximo de oxigênio e sim, pelo prejuízo à performance que a privação do sono causa aos indivíduos que possuem turnos de trabalho invertidos. Hossain et al. (2004)(22), estudando a qualidade do sono, atenção e performance das atividades realizadas por mineradores que trabalhavam em turno diurno e noturno, relataram em redução na atenção e desempenho daqueles com sono vigília invertido, onde o fator fadiga foi primordial para esta queda na habilidade e capacidade mental e motora.

\section{CONCLUSÃO}

Desta forma, podemos concluir que a inversão nos turnos de trabaIho, afetando o ciclo sono-vigília, altera não apenas o ciclo circadiano das variáveis cardiovasculares no repouso e na recuperação do esforço, como também traz prejuízos à capacidade funcional, podendo comprometer o desempenho das atividades ocupacionais.

Em razão do aumento do trabalho noturno e de seus efeitos na qualidade do sono e na saúde, é necessário um esforço contínuo dos pesquisadores e profissionais da saúde, a fim de conhecer e encontrar formas de minimizar as repercussões destas alterações sobre o organismo, conscientizando empregados e empregadores das conseqüências fisiológicas para a qualidade de vida e estado geral do indivíduo.

Todos os autores declararam não haver qualquer potencial conflito de interesses referente a este artigo.

\section{REFERÊNCIAS BIBLIOGRÁFICAS}

1. Stemberg H, Rosenthal T, Shamiss A, Green M. Altered circadian rhythm of blood pressure in shift workers. J Hum Hypertens 1995; 9: 349-53.

2. Giles T. Relevance of blood pressure variation in the circadian onset of cardiovascular events. J Hypertens. 2005; 23: S35-9.

3. Furlan R, Barbic F, Piazza S, Tinelli M, Seghizzi P, Malliani A. Modifications of Cardiac Autonomic Profile Associated With a Shift Schedule of Work. Circulation 2000; 102: $1912-6$.

4. Rotenberg L, Portela L F, Marcondes W B, Moreno C, Nascimento C P. Gênero e trabalho noturno: sono, cotidiano e vivência de quem troca a noite pelo dia. Cad Saúde Pública 2001; 3, $639-49$.

5. Marques N, Araujo J F. Ampliação dos conhecimentos em cronobiologia e sono. Ciência Hoje 2001; 30: $41-3$.

6. Collado-Ardon R, Aguilar R, Álvarez-Gayou J L, Serrano C C, Kuri P, Martín Del Campo A, Nava R, Pérez I, Valdés P, Vera A. El cambio de horario y la salud. Rev Facul Med - UNAM. 2001; 44. Disponível em:< http://www.facmed.unam.mx/publicaciones/revista/Un15-cambio.htm> Acesso em: 03 fev. 2004.

7. Sevilla-Martínes N. Ritmos Circadianos y riesgo en el trabajo nocturno. Cataluña. [s.n., 199-]: 34-6.

8. Gaspar S, Moreno C, Menna-Barreto L. Os plantões médicos, o sono e a ritmicidade biológica. Rev Ass Med Brasil 1998; 44: 239-45.

9. Reilly T. Human Circadian rhythms and exercise. Crit Rev Biomed Eng 1990, 18: 165-80

10. Almondes KM, Araújo J F. Padrão do ciclo sono-vigília e sua relação com a ansiedade em estudantes universitários. Estudos de Psicologia 2003; 8: 37-43.

11. Di Nocera F, Fabrizi R, Terenzi M, Ferlazzo F. Procedural errors in air traffic control: effects of traffic density, expertise, and automation. Aviat Space Environ Med 2006; 77: 639-43.

12. Boag C, Neal A, Loft S, Halford GS. An analysis of relational complexity in an air traffic control conflict detection task. Ergonomics 2006; 15: 1508-26.
13. White W B. Relevance of blood pressure variation in the circadian onset of cardiovascular events. J Hypert 2003; 21: S9-15.

14. Reilly T, Atkinson G, Waterhouse J. Cronobiologia e Desempenho Físico. In: Garret Jr., W. E.; Kirkendall, D. T. A Ciência do exercício e dos esportes. Porto Alegre: ArtMed, 2003: 378-400.

15. Cohen C J. Human circadian rhythms in heart rate response to a maximal exercise stress. Ergonomics 1980; 23: 591-5.

16. Reilly T. Circadian variation in ventilatory and metabolic adaptations to submaximal exercise. Br J Sports Med 1982; 16: 115-6.

17. Yamasaki F, Schwartz J E, Gerber L M, Warren K, Pickering T G. Impact of Shift Work and Race/Ethnicity on the Diurnal Rhythm of Blood Pressure and Catecholamines. Hypertension 1998; 32: 417-23.

18. Scheer F A J L, Van Montfrans G A, Van Someren E J W, Mairuhu G, Buijs R M. Daily Nighttime Melatonin Reduces Blood Pressure in Male Patients With Essential Hypertension. Hypertension 2004: 43: $192-7$.

19. Chau N P, Mallion J M, Gaudemaris R D, Ruche E, Siche J P, Pelen O, Mathern G. Twenty-four-hour ambulatory blood pressure in shift workers. Circulation 1989; 80: 341-7.

20. Deschenes M R, Sharma J V, Brittingham KT, Casa D J, Armstrong L E, Maresh C M. Chronobiological effects on exercise performance and selected physiological responses. Eur J App Physiol 1998; 77: 249-56.

21. Hildebrand G, Strempel H. Chronobiological problems of performance and adaptional capacity. Chronobiologia 1974; 4: 103-5.

22. Hossain J L, Reinish L W. Heslegrave R J, Hall G W, Kayumov L, Chung S A, et al. Subjective and Objective Evaluation of Sleep and Performance in Daytime Versus Nighttime Sleep in Extended-Hours Shift-Workers at an Underground Mine. J Occup Envirom Med 2004; 46: 212-26. 